wall thickness, systolic function and contractile reserve. A subgroup of segments, however, fail to recover normal perfusion, with deleterious effects on function.

Original article BodíV et al. (2006) Microvascular perfusion one week and six months after myocardial infarction by first-pass perfusion imaging CMR. Heart [doi: 10.1136/ hrt.2005.077305]

\section{Cardiovascular risk markers reduced in individuals who follow a Mediterranean diet}

Accumulating evidence suggests that adherence to a Mediterranean-style diet, a diet low in saturated fat and high in monounsaturated fat and dietary fiber, reduces the risk of cardiovascular disease. To examine the short-term effects of Mediterranean dietary habits on intermediate markers of cardiovascular risk, a multicenter trial was carried out in Spain.

The investigators allocated 772 adults, at high risk of cardiovascular disease, to either a lowfat diet or one of two Mediterranean-style diets. Those assigned to a Mediterranean-style diet were given either 1 l of virgin olive oil per week or $30 \mathrm{~g}$ of nuts per day. Both Mediterranean diet groups received personalized dietary advice, while those allocated to the low-fat diet were given a leaflet listing dietary recommendations. Changes in cardiovascular risk markers from baseline levels were examined at a 3-month follow-up visit.

Compared with participants in the low-fat diet group, those following a Mediterraneanstyle diet showed a decrease in systolic blood pressure, lower fasting blood glucose levels, and a reduction in the plasma concentration of several inflammatory biomarkers at follow-up. Those in the Mediterranean diet groups had an improved lipid profile after 3 months; lipid profile did not change in the low-fat diet group. The nut-supplemented Mediterranean diet had a triglyceride-lowering effect. Nondiabetic individuals following the Mediterranean-style diets recorded lower fasting insulin levels and reduced insulin resistance compared with similar participants in the low-fat diet group.

The authors suggest that the cardiovascular benefits of Mediterranean dietary habits might be related to changes in lipid profiles, and to reductions in blood pressure, inflammatory status and insulin resistance.

Original article Estruch R et al. (2006) Effects of a Mediterranean-style diet on cardiovascular risk factors: a randomized trial. Ann Intern Med 145: 1-11

\section{First-trimester exposure to ACE inhibitors is associated with congenital malformations}

Women should not be given angiotensinconverting-enzyme (ACE) inhibitors during the second and third trimesters of pregnancy, as these drugs have been linked with an increased risk of fetopathy. Data on the use of ACE inhibitors in the first trimester are sparse, however, and researchers in the US have addressed this issue using data from 29,507 births recorded in the Tennessee Medicaid database.

In total, 411 infants had been exposed to antihypertensive medications during the first trimester alone; 209 of these had been exposed to ACE inhibitors and 202 to other hypertensive medications. Overall, 856 infants were diagnosed with at least one major congenital malformation not related to a chromosomal defect or a clinical genetic syndrome. The most common types of malformation were cardiovascular, musculoskeletal and gastrointestinal malformations, affecting 305, 195 and 119 infants, respectively. The risk of major congenital malformation was over twofold higher in infants who had been exposed to ACE inhibitors in the first trimester alone than in those not exposed to antihypertensive medications (risk ratio 2.71). This increased risk results mainly from higher risks of cardiovascular and central nervous system malformations (risk ratios 3.72 and 4.39, respectively); other malformations were not significantly higher in infants exposed to ACE inhibitors. Exposure to antihypertensive medications other than ACE inhibitors in the first trimester had no effect on the risk of any major congenital malformation.

The authors conclude that ACE inhibitors are associated with an increased risk of a major congenital malformation and should not be used during pregnancy.

Original article Cooper WO et al. (2006) Major congenital malformations after first-trimester exposure to ACE inhibitors. N Engl J Med 354: 2443-2451 